\title{
Formación en Trabajo Social con conciencia global y compromiso local: un caso de buena práctica educativa
}

\author{
Training global awareness and local engagement in Social Work: \\ a case of good educational practice
}

\author{
María José AguiLAR IDÁÑEZ \\ Universidad de Castilla-La Mancha \\ mariajose.aguilar@uclm.es \\ Daniel BURASCHI \\ Universidad de Castilla-La Mancha \\ buraschidaniel@hotmail.com
}

Recibido: 29/04/2014

Revisado: 14/05/2014

Aceptado: 17/09/2014

Disponible on line: 10/12/2014

\begin{abstract}
Resumen
Analizamos una experiencia formativa que ha sido considerada por la IASSW como ejemplo de buena práctica educativa internacional en Trabajo Social. Se trata de un programa on-line de formación de postgrado para trabajadores sociales y otros profesionales de la intervención social que está basado en el análisis crítico de modelos implícitos (no conscientes y frecuentemente etnocéntricos) y el desarrollo de competencias interculturales (mindset and skillset). El programa forma a profesionales globalmente conscientes y localmente comprometidos con la defensa activa de las minorías y grupos vulnerables, capaces de implementar procesos de empoderamiento a nivel individual y colectivo. El Modelo de Intervención Social Intercultural (MISI) es el eje central (teórico y práctico) del programa formativo. Se trata de un modelo que utiliza y se inspira en otros modelos teóricos del Norte y del Sur: anti-opresivos, de potenciación y defensa, modelo de concientización, modelo de mediación trasformativa; aunque adaptados a realidades multiculturales con mayor complejidad y diversidad que aquellas en que se desarrollaron los modelos citados, y con un componente aplicado mucho más claro que los anteriores. Académicamente, el programa es innovador porque está dirigido a estudiantes internacionales (hasta ahora han participado personas de 21 países), incluye formación de grado y posgrado, y los profesores tienen perfiles formativos de origen muy diversos y con experiencia práctica en varios países del norte y del sur (Europa y América Latina). Las prácticas se realizan en diferentes países, siempre en contextos multiétnicos y culturalmente diversos. Es el único programa de formación en lengua española de estas características, y los egresados tienen un porcentaje de inserción laboral muy elevado. En el texto se describen los fundamentos teórico-conceptuales y aspectos pedagógicos relevantes que pueden ser de aplicabilidad en otros contextos.
\end{abstract}

Palabras clave: Trabajo Social, postgrado, investigación-acción, modelos implícitos, modelo de intervención social intercultural (MISI).

\begin{abstract}
We analyze a learning experience that has been considered by the IASSW as an example of good practice international in social work education. It is a postgraduate online training program for social workers and other practitioners of social intervention, based on the critical analysis of implicit models (non-conscious and often ethnocentric) and the development of intercultural skills (mindset and skill set). The program train professionals globally aware and locally committed to the advocacy of minorities and vulnerable groups, able to implement processes of empowerment at individual and collective level program. The Model of Intercultural Social Intervention (MISI) is the central axis (theoretical and practical) of the educational program. The MISI is used and is inspired by other practice theoretical models, theories and perspectives from the South (mainly) and the North: consciousness raising model, antioppressive, empowerment and advocacy, transformative mediation model, etc.; though adapted to multicultural realities with major complexity and diversity than those in which the mentioned models developed, and with an applied component clearer than the previous ones. Academically, the program is innovative as it is for international students (so far have involved people from 21 countries), including graduate and postgraduate students, and teachers have different training profiles with different origin and practical experience in several countries of the North and of the South (Europe and Latin America). Practices are held in different countries, always in multiethnic and culturally diverse contexts. It is the only social work education program in Spanish of these characteristics, and our graduates and the graduates have a very high percentage of employment. Relevant theoretical and pedagogic aspects that can be applied in other contexts are described in this text.
\end{abstract}

Keywords: Social Work, master degree, action-research, implicit models, intercultural social intervention model. 
Referencia normalizada: Aguilar Idáñez, M.J., y Buraschi, D. (2014): «Formación en Trabajo Social con conciencia global y compromiso local: un caso de buena práctica educativa». Cuadernos de Trabajo Social, 27(2): 279-289.

Sumario: Introducción. 1. Fundamentos del programa educativo. 2. Algunos ejes centrales teóricos y prácticos. 3. Conclusiones. 4. Referencias bibliográficas.

\section{Introducción}

Estamos convencidos de que nuestra visión del mundo no es el mundo, y de que no se puede resolver un problema con la misma mentalidad que se ha creado. Por ello, el postgrado forma a trabajadores sociales conscientes globalmente y comprometidos localmente con la defensa de las minorías y los grupos vulnerables, capaces de implementar procesos de empoderamiento, tanto a nivel individual como colectivo, en diferentes países y diferentes contextos multiculturales. Se presentan en este artículo los fundamentos del programa educativo y sus principales ejes teóricos y prácticos, centrándonos en los aspectos que pueden ser replicables en otros contextos ${ }^{1}$.

\section{Fundamentos del programa educativo}

\subsection{Las razones del programa formativo: las migraciones y la diversidad cultural son nue- vas realidades que ofrecen nuevas oportuni- dades para la formación en Trabajo Social}

La diversidad cultural constituye un asunto central y de importancia creciente en las sociedades actuales. El pluralismo cultural, por lo tanto, es un hecho que representa para el Trabajo Social nuevos retos y oportunidades. Retos prácticos de orden intelectual, emocional, político y ético, y retos técnicos específicos. Pero la migración también es una realidad que nos ofrece nuevas oportunidades a los trabajadores sociales, ya que la diversidad cultural abre nuevas posibilidades de acción profesional y nos obliga a repensar y reformular críticamente las tradicionales formas de intervención. Esta circunstancia, además, nos ayuda y obliga a reflexionar profundamente sobre la praxis profesional y nues- tros marcos de acción y pensamiento. Nos confronta con el compromiso de nuestra misión profesional, valores y principios propios del Trabajo Social.

Como consecuencia de la globalización, los trabajadores sociales estamos inmersos en procesos constantes de cambio, y el hecho migratorio, junto con el desafío intercultural que implica, nos impone la necesidad de un reciclaje y una formación continua, en aspectos que tradicionalmente no se consideran en el curriculum. El compromiso con los más desfavorecidos y excluidos es un elemento inherente a la profesión, y el desafío intercultural nos puede ayudar a reivindicarlo en estos momentos de fuertes presiones contra el Estado de bienestar, de cierta desorientación sobre nuestro rol profesional y de pérdida de iniciativa en muchas intervenciones sociales, políticas, universitarias, etc. Por ello, los procedimientos tradicionales, las herramientas, los marcos teóricos, la formación, los recursos y el modo de concebir la atención profesional en los servicios sociales, ya no sirven ni son eficaces.

Son numerosos los autores y expertos que, en diferentes países, hemos advertido la necesidad de una nueva formación profesional, a la que nuestro programa proporciona una respuesta efectiva (Ronnau, 1994; Legault, 1997; Verbunt, 1994, 1999 y 2004; Lévesque, 2004 y 2006; Fook, 2004 y 2012; Aguilar, 2004 a 2013; Aguilar y Buraschi, 2012; Payne y Askeland, 2008; Novak y Van Ewijk, 2010) entre otros. Consideramos que es necesario desarrollar tanto nuevos conocimientos y habilidades como una sensibilización personal y actitudes nuevas en el modo de ser y actuar profesional. Se trata

${ }^{1}$ Es un posgrado on-line de la Universidad de de Castilla-La Mancha (España), que se inició en 2004. Por la inevitable limitación de espacio no se incluimos información detallada sobre algunos aspectos académicos concretos del programa, que pueden ser consultados ampliamente en: www.mii.posgrado.culm.es. En este artículo no se hará referencia, por lo tanto, a la descripción del programa (disponible en internet) ni a sus resultados detallados, publicados enwww.mii.posgrado.uclm.es. 
de un proceso educativo donde es imprescindible articular una actitud intelectual de apertura al otro con un trabajo de autoanálisis y conocimiento de uno mismo, integrando elementos cognitivos y afectivos.

\subsection{Objetivo y enfoque: profesionales cons- cientes globalmente y comprometidos local- mente en perspectiva intercultural crítico- transformadora}

El objetivo del programa es formar a profesionales con conciencia global de los problemas y con un compromiso local en su entorno, centrado en la defensa activa de las minorías y los grupos más vulnerables, que sean capaces de implementar procesos de empoderamiento individual y colectivo. Para lograrlo el programa educativo ofrece capacitación y formación axiológica, teórica, metodológica y práctica en materia de intervención profesional con inmigrantes (particularmente en el campo de la mediación intercultural, aunque no exclusivamente), así como conocimientos, habilidades y destrezas en investigación aplicada al ámbito de las migraciones internacionales y los estudios interculturales. Todo ello, con una perspectiva interdisciplinar de la intervención social y con un enfoque intercultural crítico-transformador.

Los modos de intervención social dominantes son un reflejo de las diversas formas de respuesta que suelen darse a la gestión de la diversidad en nuestras sociedades; y que han resultado ser poco eficaces en el terreno de la convivencia ciudadana para una ciudadanía inclusiva. Son tres los modos de respuesta más frecuentes (Aguilar y Buraschi, 2012a):

- Asimilación subalterna: un modelo basado en la absorción de las minorías étnico-culturales, pero que ocupan una posición subalterna en la estructura social.

- Racismo culturalista: una perspectiva que racializa las diferencias culturales, reduciendo la complejidad de las personas a una única categoría.

- Estética intercultural: consiste en una celebración superficial de la diversidad cultural más parecida al exotismo colonial que a la perspectiva intercultural.

Las limitaciones y carencias de estas tres respuestas imposibilitan y dificultan la cons- trucción de una ciudadanía inclusiva, y una convivencia intercultural transformadora y superadora de las desigualdades y asimetrías de poder entre grupos sociales. Es necesario desarrollar una respuesta interculturalista crítica y transformadora, es decir, una alternativa válida para superar esas barreras, desigualdades y asimetrías que subyacen en las tres respuestas señaladas. El interculturalismo es un tipo de respuesta normativa al hecho de la pluralidad cultural, que se basa en una efectiva convivencia, aprendizaje y enriquecimiento mutuos. Este enfoque se diferencia y se opone a otras respuestas normativas tales como la «asimilación» de las culturas minoritarias por parte de la cultura mayoritaria, y también a la mera «tolerancia mutua» de cierto multiculturalismo («iguales, pero separados»). El enfoque intercultural que proponemos es crítico porque implica la deconstrucción del asimilacionismo subalterno y del racismo culturalista además de la superación de ciertas formas reduccionistas de entender el interculturalismo; $\mathrm{y}$ es transformador porque supone repensar nuestra forma de concebir la identidad, la cultura, la participación y la ciudadanía.

Nuestro enfoque crítico-transformador del interculturalismo no se limita al reconocimiento de la diferencia y a la promoción de la interacción positiva entre personas o grupos con diferentes horizontes culturales de referencia, sino que apuesta por la lucha contra las desigualdades sociales, económicas y políticas; y contra las discriminaciones étnicas, raciales y culturales.

\section{Algunos ejes centrales teóricos y prácticos}

\subsection{Modelos implícitos, etnocentrismo y pre- juicio en los trabajadores sociales}

La implementación de las políticas sociales destinadas a la integración de las personas migrantes depende en buena medida de la práctica profesional. Los trabajadores sociales hemos sido socializados profesionalmente de acuerdo con unos modelos de intervención monoculturales y nordoccidentales de tipo clínico y terapéutico, que no se evidencian como los más apropiados para abordar la intervención en las nuevas realidades de diversidad cultural. En definitiva, no todos los modelos teóricos y metodológicos de intervención profesional en el ámbito social son adecuados ni oportunos ni pertinentes, si los analizamos en perspectiva intercultural y bajo el 
prisma de la construcción de una ciudadanía inclusiva.

Debemos diferenciar claramente entre un modelo explícito de intervención y un modelo implícito. Un modelo explícito de intervención social es un conjunto reflexivo y coherente de pensamientos y conceptos referidos a principios, teorías, estrategias y acciones construidas basándose en unas categorías de población que dibujan una guía de intervención social en relación con una problemática concreta. Los modelos implícitos, por su parte, son un marco de referencia, una construcción simplificada $\mathrm{y}$ esquemática de la realidad, que aportan una explicación de la misma y que conforman un esquema general referencial que guía la práctica, de forma irreflexiva.

Aunque nos resulte incómodo, tenemos que tomar conciencia de que, con frecuencia, nuestra forma de trabajar con y para las personas migrantes se basa en valores, supuestos y estereotipos que pueden reproducir nuevas formas de racismo y que nos impiden reconocer las reales necesidades de las personas.

En la base de nuestras formas de diseñar programas de intervención, de nuestra forma de comprender los problemas sociales, de nuestras prácticas de trabajo cotidiano con las personas inmigrantes, se encuentran modelos implícitos, que son el marco a través del cual interpretamos, comprendemos y actuamos. Reproducen de forma sistemática una acción específica con los inmigrantes y sus problemáticas, y una específica visión de dichas personas, de su contexto, de sus recursos y de sus problemas. En muchas ocasiones se trata de modelos basados en esquemas universalizantes que parten de conceptos particulares o que exacerban las diferencias culturales. No debemos olvidar que la forma de encuadrar un problema determina la de resolverlo y que, en no pocos casos, la mayor dificultad para una eficaz intervención social estriba en un mal encuadre del problema (falso, distorsionado, erróneo, reduccionista o sesgado) que nos imposibilita e impide hallar su correcta solución.

Hemos afirmado que un modelo de intervención es un conjunto coherente de pensamientos y conceptos referidos a teorías, sentimientos, actitudes y acciones construidas en base a unas categorías de población, que dibujan una guía de intervención social en relación con una problemática concreta. Los modelos son, por lo tanto, una construcción simplificada y esquemática de la realidad, que aportan una explicación de la misma y que conforma un esquema general referencial que guía la práctica. Los modelos implícitos dependen y se configuran a partir de la interrelación de varios elementos: cómo se define la situación problema que se pretende modificar; qué intereses están en juego y que a menudo van mucho más allá de los objetivos declarados en la intervención; cuáles son los valores dominantes; cuáles las estrategias que se consideran más legítimas para enfrentar el problema; cómo se definen a las personas involucradas, en particular qué roles y estatus se les asignan y qué relación se considera que deberían tener entre ellas.

Estos elementos reflejan los valores, las creencias y los prejuicios del profesional; y de estos elementos y sus interrelaciones derivan tanto la naturaleza específica de la relación que se establece entre el agente social y el «usuario» inmigrante, como las formas concretas y operativas de esa intervención profesional (orientación metodológica, procedimientos, etc.). Son muy pocos los profesionales conscientes de la presencia de estos modelos implícitos, ya que, de ordinario, sólo se abordan en la formación y el ejercicio profesional los modelos explícitos, es decir, los modelos teóricos que constituyen el marco y encuadre profesional deliberadamente elegido por el agente profesional, que opta así por unas teorías u otras a la hora de orientar su praxis profesional. La incoherencia y contradicción entre el modelo explícito y el modelo implícito son muy frecuentes, por cuanto el implícito se suele mantenerse a nivel inconsciente.

\subsection{El culturalismo etnocéntrico como pro- ceso básico de los modelos implícitos}

La lógica que está detrás de los modelos dominantes en la intervención social es un proceso que denominamos «culturalismo etnocéntrico». Se trata de un proceso de construcción social de la alteridad basado en categorías rígidas, etnocéntricas, esencialistas e impuestas a las personas migrantes. El culturalismo etnocéntrico se compone de tres elementos íntimamente relacionados: la categorización impuesta, el etnocentrismo y el culturalismo (Aguilar y Buraschi, 2012b).

La categorización impuesta. Es la construcción de un sistema de clasificación que tiene el 
poder de reproducir y crear lo que, aparentemente, se limita a describir. La forma de pensar y categorizar a las personas migrantes condiciona el estilo de intervención. No hay que olvidar que el discurso institucional y de los trabajadores sociales es dominante y legítimo y que suele prevalecer sobre las definiciones de las propias personas migrantes, en este sentido tiene el poder de normalizar y naturalizar categorías arbitrarias. Son tres grupos de metáforas las que se utilizan más para categorizar a las personas migrantes: las metáforas que definen a la persona migrante como víctima; las metáforas que le definen como amenaza; y las metáforas que categorizan a la persona migrante como carenciada.

- La categoría de víctima es la que define a estas personas como sujetos vulnerables, pasivos, indefensos e incapaces de enfrentarse a los problemas y de ser dueños del propio proyecto migratorio.

- La categoría de amenaza hace hincapié en el peligro que determinadas personas migrantes pueden representar para nuestros valores, nuestras creencias o para el Estado de bienestar. Se concibe a las personas migrantes principalmente como un problema.

- La visión de la persona migrante como carenciada se basa en una visión etnocéntrica de lo que consideramos «normal», se centra en las supuestas carencias culturales, sociales, económicas, lingüísticas, etc.

La categorización implica un proceso de reducción de la complejidad de la persona migrante a una o pocas de sus supuestas características. Según este punto de vista, los modelos implícitos son un dispositivo de reducción y de invisibilización de parte de la realidad social. A menudo no tenemos en cuenta la historia previa, focalizamos nuestra atención en los problemas y no en la capacidad de las personas para hacer frente a sus dificultades; invisibilizamos determinados factores sociales y coyunturales, las múltiples identidades y la complejidad cultural. De esta forma la persona migrante pierde la posibilidad de autodefinirse, la definición de su identidad no le pertenece. La indiferencia y la exclusión son formas sutiles de racismo, porque niegan la complejidad del sujeto, e incluso le niegan la consideración de tal, para convertirlo en un mero objeto.
La otra característica del proceso «culturalista etnocéntrico» es que solemos aplicar nuestras categorías analíticas a otras realidades sociales, olvidando que existen diferencias: adaptamos la realidad a las categorías en lugar de adaptar las categorías a la realidad. Es decir, creemos que las categorías que utilizamos en nuestro trabajo son universales y se puedan aplicar en todos los contextos, y es falso.

Finalmente el último elemento del proceso «culturalista etnocéntrico» es la exageración de los factores culturales y la esencialización de la cultura. Interpretamos el comportamiento de las personas únicamente por su pertenencia (real o supuesta) a una determinada cultura, confundiendo las diferencias sociales con las diferencias culturales. De este modo se oculta la incapacidad (y falta de voluntad) del Estado para resolver satisfactoriamente los problemas, y se utilizan las diferencias culturales como pantalla para tapar los debates que la sociedad no quiere afrontar.

Esta tendencia se nota especialmente en la fase de diagnóstico y análisis de los problemas de integración: la delincuencia, el fracaso escolar, la pobreza se explican a través de variables culturales como los factores religiosos, la «mentalidad», la «orientación hacia el presente», el «fatalismo», etc. Este modelo de intervención tiende a sublimar la pobreza, subestima los factores económicos y sociales y sobrestima los factores culturales. El diagnóstico se sigue realizando como un peritaje exterior a los interesados, a quienes no se les consulta ni se les invita a participar activamente en el mismo. Con frecuencia se formulan proyectos de intervención sin tomar en consideración la participación de los propios interesados, con lo que la potencial eficacia de las intervenciones se ve muy reducida. En otros casos, se distorsiona la visión del inmigrante, al que se clasifica como «bueno» o «malo» en función de su mayor o menor proximidad con las normas sociales, interviniendo desde modelos clínicos que tienden a interpretar su situación de marginación como inadaptación, falta de voluntad o desvío que hay que corregir, «normalizando» sus comportamientos.

\subsection{El modelo de intervención social inter- cultural}

Además del análisis crítico de los modelos implícitos en la práctica profesional, el programa 
formativo aplica el enfoque intercultural crítico y transformador a la intervención social, desarrollando pautas para la construcción de un modelo de intervención social intercultural (Aguilar, 2004, 2006, 2008, 2010, 2011a, 2011b, 2012; Aguilar y Buraschi, 2012a, 2012b, 2013), basado en la comprensión profunda del otro, en el desarrollo de la empatía intercultural, y en la adquisición de competencias interculturales. La intervención social intercultural no trata de incluir nuevas herramientas de intervención, sino de trasformar nuestra forma de pensar y vivir la diversidad cultural, integrando aspectos cognitivos y afectivos.

Hay que tener en cuenta que la mayoría de los profesionales hemos sido socializados en horizontes culturales monoculturales y etnocéntricos y, a menudo, no tenemos las herramientas para gestionar la incertidumbre y el estrés que genera la relación con personas cuyos comportamientos no logramos comprender ni prever. La incomprensión es doble: no sabemos interpretar los comportamientos ajenos (incertidumbre explicativa) y no logramos prever las posibles reacciones (incertidumbre predictiva). Esto significa que, para trabajar eficazmente en un espacio multicultural, no son suficientes la buena voluntad y las actitudes positivas hacia la diversidad; es necesario tener las competencias para comprender la diversidad, gestionar los conflictos y relacionarse eficazmente. Nuestros valores y creencias pueden no ser suficientes cuando nos encontramos en situaciones complejas y ambiguas. Los valores y las creencias tienen que ir acompañados con conocimientos, habilidades, actitudes y destrezas que nos permitan manejar eficazmente las situaciones conflictivas o ambiguas. Sin estas competencias el encuentro intercultural se puede transformar en choque cultural y alimentar el racismo y la xenofobia.

El modelo explícito de intervención social intercultural se formula con análisis teóricos sobre las formas dominantes del discurso y el racismo de las élites (Van Dijk, 2000), incluyendo el racismo institucional puesto en práctica desde las políticas e intervenciones sociales (Rivera, 2009; Aguilar, 2011b). Tiene otras referen- cias teóricas en las propuestas de Trabajo Social antiopresivo (inicialmente elaboradas por Bárbara B. Solomon, 1976) ${ }^{2}$; en los conceptos de empowerment y advocacy (Rose y Black, 1985), reconocimiento (Honneth, 1997), interseccionalidad (Crenshaw, 1995), en la sociología reflexiva de Bourdieu (1980), la antropología crítica (Marcus y Fisher, 1986; Rosaldo, 1993), el construccionismo social (Berger y Luckman, 2001), y en la filosofía de la alteridad de Lévinas (1991).

Desde el punto de vista de la operatividad del modelo, las inspiraciones teóricas y prácticas más relevantes son:

a) El enfoque de concientización y liberación, que se inicia en América Latina con las obras de Paulo Freire, particularmente sus propuestas de «educación problematizadora»y «dialogicidad» (Freire, 1970, 1992 y 1996). Estas propuestas se aplican también en el teatro del oprimido de Boal (1978) —en sus versiones latinoamericanas y europeas - así como en diferentes programas latinoamericanos de «educación popular», particularmente los promovidos por el Consejo de Educación de Adultos de América Latina (Kaplún (1983, 1985; Núñez, 1989).

b) El enfoque comunicacional sistémico de la escuela de Palo Alto (Watzlawick, Beavin, y Jackson, 1967).

c) La pedagogía no-violenta italiana de Capitini (1968) y Dolci (1996).

d) La mediación transformativa propuesta por Bush y Folger (1994) y sus conceptos de revalorización y legitimación.

e) Las aportaciones de la psicología social aplicada y de la psicología cultural (Bruner, 1991).

En este modelo el trabajador social utiliza las estrategias propias del empoderamiento para reducir, eliminar, combatir o invertir las valoraciones negativas que se plantean, desde el conjunto de la sociedad en general y desde el poder y sus grupos en particular, sobre los inmigrantes. El empleo y fortalecimiento de redes de apoyo mutuo, la utilización de la capaci-

2 Las propuestas actuales de Trabajo Social crítico y antidiscriminatorio más relacionadas con nuestro modelo son, entre otras: Okitikpi y Aymer (2010), Bhatti-Sinclair (2011), Lavalette y Ioakimidis (2011), Langan (2011), Baldwin (2011), y Fook (2012). 
tación como transferencia de saberes, habilidades y tecnologías, capacidad para tomar decisiones y organizarse, de interpretar, proyectar y actuar colectivamente, etc. son algunos ejemplos de estas estrategias, donde no se niega el conflicto sino que se trabaja con él y desde él cuando es preciso. Este modelo exige un compromiso para mantener servicios socioeducativos y programas de intervención social efectivamente igualitarios y para enfrentarse a valoraciones negativas fuertemente arraigadas, incluso en la cultura técnico-profesional e institucional.

Se trata de implementar procesos de diálogo, comprensión y mejora, utilizando conceptos, técnicas y estrategias propias del Trabajo Social emancipatorio y radical para fomentar la mejora y la autodeterminación de los participantes. Es decir, para el desarrollo de habilidades que permitan a las personas, organizaciones y comunidades mejorar por sí mismos sus actuaciones, y favorecer el cambio social necesario para que las situaciones resulten más justas y equitativas.

En esta perspectiva, el trabajador social tiene un papel muy diferente y diverso: frente al clásico papel de experto gestor y organizador, en este enfoque puede ser un facilitador, un colaborador, un defensor, un mediador o un formador, dependiendo de las dinámicas generadas por el proceso de intervención. Nuestra acción se convierte así en un instrumento pedagógico y político de fortalecimiento emancipador de organizaciones, personas y grupos. El concepto de autodeterminación es un fundamento básico de este modelo, que se define como un conjunto de habilidades interrelacionadas, tales como: habilidad para identificar y expresar necesidades; establecer objetivos o expectativas y trazar un plan de acción para alcanzarlas; identificar recursos; hacer elecciones racionales entre cursos de acción alternativos; desarrollar actitudes apropiadas para conseguir los objetivos; evaluar resultados, etc.

La intervención social con inmigrantes encaminada a la autodeterminación si es llevada a cabo en contextos multiculturales necesita, previamente, un proceso de capacitación intercultural de los profesionales, para evitar que la intervención social se trasforme, aunque sea de forma involuntaria, en un dispositivo de reproducción de las desigualdades sociales.

\subsection{Competencias interculturales en el mo- delo de intervención social intercultural}

Con este modelo de intervención proponemos la adquisición de un conjunto de competencias interculturales en Trabajo Social, basado en las aportaciones de Chen y Starosta (1996), Byram (1997), Aneas (2003) y Sclavi (2003). Entendemos las competencias interculturales como un conjunto de conocimientos, actitudes y destrezas que nos permiten trabajar en contextos multiculturales de forma eficaz contribuyendo a la convivencia intercultural. No se trata solamente de comportarse de forma pertinente y adaptarse a los diferentes contextos, sino de transformar las relaciones para contribuir a transformar la sociedad.

Siguiendo la propuesta de Milton Bennett (1986), diferenciamos dos dimensiones de competencias interculturales: la primera hace referencia a una manera de mirar al mundo, incluye los aspectos cognitivos, emocionales y actitudinales que son transversales a todas las competencias específicas y que conforman lo que podríamos denominar la mente intercultural: tolerancia a la ambigüedad, apertura y curiosidad hacia la diversidad, flexibilidad mental, creatividad, entre otras. La segunda hace referencia a todos los aspectos comportamentales y a las capacidades específicas y las estrategias que se necesitan para trabajar eficazmente con personas y grupos con diferentes referentes culturales. Según nuestro modelo, las competencias específicas de los profesionales que trabajan en contextos multiculturales son: la concientización intercultural; la comprensión de otros marcos culturales de referencia; la sensibilización intercultural; la asertividad intercultural y la gestión creativa de los conflictos.

Concientización intercultural. Paulo Freire formuló el concepto de concientización para describir el proceso de transformación personal y social que protagonizan las personas oprimidas cuando toman conciencia de la lógica de opresión que sustenta las relaciones de poder en las cuales están implicadas. El aspecto interesante del concepto de concientización es que no hace referencia solamente al contexto de dominación, sino también a los modelos de opresión que estructuran la mente de los oprimidos. Podemos aplicar este concepto a las relaciones entre las personas en contextos multiculturales y 
hablar de concientización intercultural como la toma de conciencia de nuestros presupuestos implícitos, de las creencias, valores implícitos que, a menudo de forma automática, influyen en nuestras interpretaciones del mundo y en nuestros comportamientos. Como hemos visto en la primera parte, estos modelos implícitos son a menudo las barreras invisibles que nos impiden comprender otros marcos de referencia, comunicarnos eficazmente, gestionar conflictos y reproducen estereotipos, prejuicios y relaciones desiguales.

La conciencia del propio horizonte cultural en general, y de los modelos implícitos que guían nuestra intervención social en particular, es el primer paso para el desarrollo de las competencias interculturales puesto que «es nuestra mirada la que muchas veces encierra a los demás en sus pertenencias más limitadas, y es también nuestra mirada la que puede liberarlos» (Maalouf, 1998, p. 7).

La comprensión de otros marcos culturales de referencia incluye las actitudes, conocimientos y destrezas que nos permiten entender a personas que no comparten nuestro mismo marco de referencia y situaciones de contacto en contextos pluriculturales. Se basa en la capacidad de investigar la diversidad a través de «mapas dinámicos», un conjunto de claves hermenéuticas que representan una guía flexible hacia la comprensión y nos permiten cambiar nuestra forma de identificar un problema. En este sentido es una competencia fundamental en la labor de diagnóstico del trabajador social. El punto de partida no tiene que ser solamente el análisis de los problemas, sino de las competencias de las personas migrantes, sus redes, su capital social y cultural, su resiliencia. Que la pregunta fundamental de nuestro diagnóstico no sea «¿cuáles son los factores que determinan la vulnerabilidad de las personas migrantes?», sino «¿cuáles son los factores que en muchos casos han sido determinantes para las historias de éxito de estas personas?». Se trata de valorizar la resiliencia, la capacidad de enfrentarse a las adversidades y salir adelante, se trata de tomar en consideración la historia previa de las personas migrantes, sus prácticas, sus redes de apoyo, las características de la sociedad receptora, el ambiente, o el clima social en un determinado momento, los factores protectores, sus recursos internos y capacidades, sus oportunidades y dinamismos vitales (Aguilar, 2013).

Respecto a la dimensión transnacional y sociocomunitaria de la experiencia migratoria hay que subrayar que en la mayor parte de los casos, la intervención profesional desde los servicios sociales se desarrolla aplicando modelos diagnósticos (propios de enfoques clínicos nordoccidentales), que habitualmente tienden a etiquetar la realidad en función de las características de la oferta, sin plantearse seriamente la exploración comunitaria de las potencialidades y recursos propios.

La sensibilidad intercultural es una competencia que incluye la autoconciencia emocional, la actitud positiva hacia la diversidad y la empatía intercultural o exotopía. Por esta última entendemos una forma de empatía que sea eficaz en contextos pluriculturales. Con la empatía buscamos comprender la experiencia de la otra persona a partir de nuestro marco. Es una forma de salir de una visión narcisista, pero no de salir de una visión etnocéntrica. Cuando intentamos ponernos en los «zapatos de los otros» en realidad estamos poniendo los otros en «nuestros zapatos». Sclavi (2003) propone el concepto de exotopía, para definir el esfuerzo de reconocer la perspectiva autónoma del otro, una perspectiva con sentido propio, no reducible a la nuestra. En la empatía aislamos y descontextualizamos algunos rasgos de la experiencia del otro para comprenderla a partir de nuestro marco cultural, de esta forma en realidad no salimos de nosotros mismos, sino que proyectamos en el otro nuestra forma de sentir y vivir una experiencia. La exotopía genera otra forma de empatía, la empatía intercultural, un proceso en el cual la otra persona no juega un papel pasivo, sino activo, colabora con nosotros/as en la construcción de un sentido compartido de la experiencia afectiva. En este sentido podemos definir la «empatía intercultural» como la habilidad de hacer experiencia de aspectos de la realidad de forma diferente de cómo se haría desde nuestro marco de referencia a través de la construcción colaborativa del sentido de la experiencia afectiva.

La asertividad intercultural es la «habilidad para negociar los significados culturales y de actuar comunicativamente de una forma eficaz de acuerdo a las múltiples identidades de los participantes» (Chen y Starosta, 1996, p. 358). Ser asertivos en contextos multiculturales signi- 
fica saber identificar los propios estilos comunicativos; reconocer los estilos comunicativos de otras personas o grupos; saber crear canales de comunicación; tener la capacidad de conceptualizar, de explicarse, de presentarse de forma apropiada, de llegar a un grado aceptable de comprensión recíproca y gestionar la interacción de forma adecuada y eficaz.

Finalmente la gestión creativa de los conflictos es la capacidad de utilizar estrategias de resolución exploratorias basadas en un análisis complejo de los conflictos, la reestructuración de las relaciones (Bush y Folger, 1994) y la generación de alternativas basadas en la construcción de un nuevo marco de referencia común (Sclavi, 2003).

\subsection{Diseño pedagógico}

Decía Paulo Freire que educar no es transmitir conocimiento, sino crear las condiciones necesarias para su construcción. En base a ello, el diseño pedagógico del programa formativo permite lograr las capacidades mencionadas de análisis y de intervención intercultural críticotransformadoras, mediante una serie de elementos innovadores. A modo de ejemplo, mencionamos los siguientes:

Selección del profesorado. En el programa participan más de 40 profesores europeos y latinoamericanos. Todos ellos son especialistas de prestigio internacional en sus respectivos campos, y compartimos marcos críticos de análisis, así como un compromiso personal con movimientos y entidades sociales de lucha contra las diferentes formas de discriminación, en el país en que cada profesor reside. El perfil es interdisciplinar (trabajadores sociales, sociólogos, antropólogos, pedagogos, juristas, psicólogos, médicos, demógrafos, abogados, sociolingüistas, etc.). Muchos de ellos tienen formación en varias de estas disciplinas.

Selección de estudiantes. El programa de postgrado está abierto también a estudiantes de Grado. Pueden participar candidatos de cualquier país y de diferentes campos profesionales con incidencia social. Estos perfiles aseguran una gran diversidad cultural en el grupo de aprendizaje, interdisciplinariedad y diferentes perspectivas y experiencias vitales, que son muy importantes para asegurar el éxito del programa formativo ${ }^{3}$.

Selección y diseño de contenidos formativos. Se han diseñado de forma que el estudiante vaya adquiriendo de forma progresiva la capacidad de análisis crítico de la realidad, además de aumentar la autoconciencia de sí mismo. En todos los módulos formativos se incorporan contenidos cognitivos y afectivos, para lograr la adquisición de empatía intercultural desde el inicio del programa. Significa que los contenidos no se superponen o solapan, sino que se apoyan unos sobre otros, de manera constructiva y secuenciada. Los contenidos se actualizan de forma permanente durante todos los cursos ${ }^{4}$. En los foros de discusión on line participan también estudiantes egresados del programa en cursos anteriores y todos los profesores.

Secuenciación de contenidos. En el programa no hay asignaturas, sino módulos secuenciados y sucesivos de formación, que permiten un proceso de construcción del aprendizaje de tal manera que el estudiante va madurando personalmente a medida que avanza en la formación, y va tomando conciencia de sí mismo y de sus propios marcos de referencia (sobre todo implícitos). También se van adquiriendo las capacidades y actitudes propias del MISI, sobre todo desde el tercer mes de formación ${ }^{5}$.

Integración teoría y práctica. Todos los conocimientos teóricos y metodológicos se apli-

${ }^{3}$ Hasta 2013 han participado estudiantes de 21 países (Reino Unido, Francia, Italia, España, Israel, Palestina, Argelia, Marruecos, Mali, Argentina, Brasil, Bolivia, Chile, Ecuador, Perú, Colombia, Guatemala, México, República Dominicana, Haití y Estados Unidos).

${ }^{4}$ Los resultados de la evaluación de calidad que realizan los estudiantes en cada módulo y en cada curso, se utilizan para introducir mejoras en el programa de forma inmediata (muchas veces sin necesidad de esperar a que finalice el curso académico). Este rápido feedback asegura que el programa se ajuste de manera óptima a las necesidades formativas de los estudiantes.

${ }^{5}$ El programa completo dura 2 años: el primero es común a todos los estudiantes, mientras que en el segundo se elige itinerario de especialización y dentro de cada itinerario el estudiante puede diseñar «a la carta» su plan de estudios, entre los módulos optativos que se ofertan. Para evitar una extensión excesiva de este artículo no mencionamos aquí los módulos y temario detallado de los mismos, ya que dicha información está publicada en: www.mii.posgrado.uclm.es 
can a la práctica. No interesa el conocimiento por el conocimiento, sino el conocimiento orientado a la acción. Se evalúa el resultado de la integración-aplicación de los conocimientos y la reflexión crítica sobre los mismos en la práctica y en el trabajo de campo. Todos los trabajos y talleres incorporan elementos cognitivos y emocionales, como forma de desarrollar progresivamente la capacidad de empatía intercultural, la tolerancia a la ambigüedad y la apertura mental al Otro.

Estudio y análisis observacional en perspectiva empática. En la mayoría de los módulos se incluyen ejercicios de observación crítica y autocrítica de la realidad. El hecho de que todos los estudiantes internacionales residen en su propio país, favorece mucho el enriquecimiento y el intercambio de experiencias. Una de las fortalezas del programa es precisamente ésta: cada estudiante tiene la experiencia de su propio contexto, y también la experiencia directa de los otros contextos donde residen sus compañeros de clase con lo que comparte diálogo y trabajos colaborativos de estudios y solución de casos prácticos.

Estudio y solución de casos de forma cooperativa. Se utilizan diversas perspectivas, como el análisis de incidentes críticos, el aprendizaje basado en problemas, el auto-diagnóstico participativo, la planificación participativa, etc. Se fomenta la solución colaborativa de casos y situaciones, antes de la realización de los trabajos y ejercicios individuales.

Trabajo de campo. A partir del cuarto mes de formación, todos los módulos incluyen trabajos prácticos sobre el terreno que el estudiante debe hacer en su propio contexto, con la orientación tutorial de los profesores. Los tres últimos meses del programa son exclusivamente de prácticas supervisadas, que se realizan en el país y región que el estudiante decide.

Mejora continua del programa. El sistema de evaluación permanente y sistemática del pro- grama formativo, llevada a cabo por estudiantes y profesores de manera conjunta y por separado, permite la mejora inmediata de cualquier aspecto de la formación. Se realizan encuestas de calidad muy detalladas en cada módulo y en cada curso por parte de los estudiantes, cuyos resul$\operatorname{tados}^{6}$ se utilizan para introducir los cambios necesarios (ya sea que afecten a profesorado, contenidos, organización del trabajo, adaptación al entorno cultural del estudiante, etc.). Asimismo, se utilizan las valoraciones de estudiantes y profesores realizadas en foros públicos y en otros espacios de coordinación docente interna.

\section{Conclusiones}

Los modelos implícitos etnocéntricos y culturalistas tienen consecuencias importantes en la intervención social con inmigrantes: reproducen un sistema de relación social desigual y asimétrico; refuerzan la imagen de las personas migrantes como un «grupo de exclusión social» $\mathrm{y}$ obstaculizan el proceso de autonomía e integración. Las demandas de las personas migrantes y las respuestas insuficientes de los modelos de intervención tradicionales pueden generar estrés, incertidumbre y ansiedad, pero son, también, una importante ocasión para revisar nuestros métodos de intervención, una oportunidad para ser conscientes de las limitaciones de nuestros esquemas de análisis y repensar nuestros modelos de intervención.

Dado el carácter inconsciente de los modelos implícitos, es una exigencia imprescindible el desarrollo de procesos de explicitación que hagan aflorar de manera consciente dichos modelos, explicitando los mecanismos de reproducción de las lógicas de discriminación que subyacen en los mismos. La formación profesional para el trabajo social debe incorporar estas propuestas. Se trata de ser conscientes de que las categorías que consideramos neutrales están, a menudo, estrechamente relacionadas con un determinado contexto cultural, y en algunos casos

${ }^{6}$ En los últimos diez años, las valoraciones medias de las encuestas del alumnado, referidas a distintos aspectos del programa ha sido las siguientes (en una escala de 0 a 10 ): contenidos $(9,8)$; profesorado $(9,9)$; organización del trabajo $(9,4)$; interacción y comunicaciones $(9,5)$; interés y utilidad $(9,9)$. Asimismo, todos los estudiantes, sin excepción, coinciden en recomendar la formación recibida. Los principales cambios de contenidos han sido introducidos a partir de la evaluación realizada por el profesorado y la dirección del máster, considerando la experiencia acumulada en las sucesivas ediciones. El plan de estudios ha sido modificado, ampliado y mejorado en tres ocasiones, y los contenidos temáticos se actualizan cada año. Otro indicador de impacto que arroja buenos resultados es el de la inserción laboral de los estudiantes (98 por ciento). 
se basan en presupuestos etnocéntricos reduccionistas. Además, en la formación deben aprenderse nuevos modelos alternativos de intervención, adaptados y coherentes con los valores, principios y compromiso del trabajo social.

El modelo de intervención social intercultural es una propuesta teórica y práctica, sistemática y de probada eficacia, para un Trabajo Social en contextos de diversidad cultural que tenga como objetivo lograr una ciudadanía realmente inclusiva e igualitaria, acorde con los derechos humanos. Este modelo es crítico y transformador, emancipador y potenciador en la medida en que apoya y estimula a individuos y grupos a desarrollar sus habilidades volviéndose autónomos para resolver los problemas y tomar decisiones, al mismo tiempo que aboga por un cambio estructural hacia una sociedad más justa. A nivel individual los resultados pueden observarse a través de la percepción del control de la situación, la sociabilidad y los comportamientos dirigidos a la acción. En las organizaciones, los resultados pueden incluir el desarrollo de redes organizacionales, la captación de recursos y la definición de políticas. En la comunidad, los resultados pueden ser analizados por la evidencia de procesos de inclusión social, convivencia pluralista y construcción de proyectos colectivos de mejora del entorno y la calidad de vida.

El programa formativo cuyos fundamentos teóricos y conceptuales y cuyos aspectos pedagógicos relevantes se han analizado, evidencia la necesidad y pertinencia de desarrollar acciones formativas en el campo del trabajo social, superadoras de las limitaciones que la formación tradicional plantea en los nuevos y complejos contextos de diversidad cultural.

\section{Referencias bibliográficas}

Aguilar, $\mathrm{M}^{\mathrm{a}} \mathrm{J}$. (2004). Trabajo social intercultural: una aproximación al perfil del trabajador social como educador y mediador en contextos multiculturales y multiétnicos. Portularia. Revista de Trabajo Social, 4, 153-160. Disponible en: http://dialnet.unirioja.es/servlet/articulo?codigo $=860428$.

Aguilar, $\mathrm{M}^{\mathrm{a}} \mathrm{J}$. (2006). Inmigración, integración e interculturalidad. En A. Alted (dir.) De la España que emigra a la España que acoge (pp. 556-569). Madrid: Fundación Francisco Largo Caballero y Caja Duero.

Aguilar, M J. (2008). Ciudadanía Intercultural: Materiales y propuestas desde la Sociología Visual. Albacete: GIEMIC-UCLM.

Aguilar, $\mathrm{M}^{\mathrm{a}}$ J. (2010). Modelos de intervención social con inmigrantes e interculturalidad: un análisis crítico. Inguruak. Revista Vasca de Sociología y Ciencia Política, vol. Monográfico especial 'Sociedad e Innovación en el siglo XXI', febrero 2010, 77-94.

Aguilar, M J. (2011a). Ciudadanía intercultural y animación: una experiencia innovadora para el empoderamiento de colectivos inmigrantes. Journal Sociocultural Community Development and Practices, 1, 22-47. Disponible en: http://www.atps.uqam.ca/numero/n1/pdf/ATPS_AguilarIdanez_2010.pdf.

Aguilar, $\mathrm{M}^{\mathrm{a}} \mathrm{J}$. (2011b). El racismo institucional en las políticas e intervenciones sociales dirigidas a inmigrantes y algunas propuestas prácticas para evitarlo. Documentación Social, 162, 139-166.

Aguilar, $\mathrm{M}^{\mathrm{a}} \mathrm{J}$. (2012). Pensar la intervención social con personas migradas: un desafío a nuevas formas de intervención desde el trabajo social. En A. Avaria (ed.). Desafios de la migración. ¿Cómo acercarnos a las personas migradas? Miradas de y desde la investigación e intervención social (pp. 79-112). Santiago de Chile: Ediciones Universidad Santo Tomás.

Aguilar, M ${ }^{\mathrm{a}}$ J. (2013). Trabajo social. Concepto y metodología. Madrid: Paraninfo-Consejo General de Trabajo Social.

Aguilar, M ${ }^{\mathrm{a}}$ J. y Buraschi, D. (2012a). El desafío de la convivencia intercultural. REMHU. Revista Interdisciplinar da Mobilidade Humana, 38, 27-43. Disponible en: http://www.csem.org.br/remhu/index.php/remhu/article/viewFile/298/273.

Aguilar, Ma J. y Buraschi, D. (2012b). Prejuicio, etnocentrismo y racismo institucional en las políticas sociales y los profesionales de los servicios sociales que trabajan con personas migrantes. VII Congreso Migraciones Internacionales en España. Movilidad humana y diversidad social. Bilbao, 11 al 13 de abril. Recuperado de: http://bit.ly/1e5Hk8I. 
Aguilar, Ma J. y Buraschi, D. (2013). Asociacionismo e intervención social con inmigrantes. En E. Raya, $\mathrm{M}^{\mathrm{a}} \mathrm{A}$. Espadas y M. Aboussi (coords.). Inmigración y ciudadanía activa. Contribuciones sobre gobernanza participativa e inclusión social (pp. 43-68). Barcelona: Icaria

Aneas, A. (2003). Competencias interculturales transversales en la empresa: un modelo para la detección de necesidades de formación. (Tesis doctoral del Departamento de Mètodes d'Investigació i Diagnòstic en Educació. Barcelona: Universitat de Barcelona). Recuperado de: http://hdl. handle.net/2445/42451.

Baldwin. M. (2011). Resisting the EasyCare model: building a more radical, community-based, anti-authoritarian social work for the future. En: M. Lavalette (ed.), Radical social work today. Social work at the crossroads (pp. 187-204). Bristol: The Policy Press.

Bennett, M. J. (1986). A developmental approach to training intercultural sensitivity, International Journal of Intercultural Relations, 10 (2), 179-186.

Berger, P.L. y Luckmann, T. (2001). La construcción social de la realidad. Buenos Aires: Amorrortu. Bhatti-Sinclair, K. (2011). Anti-racist practice in Social Work. Basingstoke: Palgrave Macmillan.

Boal, A. (1978). Jeux pour acteurs et non-acteurs. Pratique du thêatre de l'opprimé. Paris: Maspero. [Primera edición en español,1980. Teatro del oprimido/2. Ejercicios para actores y no actores]. México: Nueva Imagen].

Bourdieu, P. (1980). Le sens pratique. París: Minuit.

Bruner, J. (1991). Actos de Sentido. Madrid: Alianza.

Bush, R. y Folger, J. (1994). The Promise of Mediation. San Francisco: Jossey-Bass.

Byram, M. (1997). Teaching and Assessing Intercultural Communicative Competence. Clevedon: Multilingual Matters.

Capitini, A. (1968), L'Educazione aperta, Firenze: NIS.

Chen, G. M., y Starosta, W. J. (1996). Intercultural communication competence: A synthesis. En R.B. Brant (ed.). Communication Yearbook 19. Thousand Oaks: Sage.

Crenshaw, K. (1995). Mapping the Margins: Interseccionality, Identity Polítics and violence Against Women of Color. En K. Crenshaw; N. Cotanda; C. Peller; K. Thomas (eds.), Critical Race Theory. The key writings that formed the movement (pp. 357-383). Nueva York: The New Press.

Dolci, D. (1996), La struttura maieutica e l'evolverci. Firenze: NIS.

Fook, J. (2004). Some Considerations on the Potential Contributions of Intercultural Social Work. Social Work \& Society, 2 (1), 83-86.

Fook, J. (2012). Social Work. A Critical Approach to Practice (2 ${ }^{\mathrm{a}}$ ed.). Londres: Sage.

Freire, P. (1970). Pedagogia del oprimido. Montevideo: Tierra Nueva.

Freire, P. (1992). Pedagogia da esperança: um reencontro com a Pedagogia do oprimido. Río de Janeiro: Paz e Terra.

Freire, P. (1996). Pedagogía da autonomía- Saberes Necessários à prática Educativa. São Paulo: Paz e Terra.

Honneth, A. (1997). La lucha por el reconocimiento: por una gramática moral de los conflictos sociales. Barcelona: Crítica.

Kaplún, M. (1983). Hacia nuevas estrategias de comunicación en la educación de adultos. Santiago de Chile: UNESCO.

Kaplún, M. (1985). El comunicador popular. Quito: CIESPAL.

Langan, M. (2011). Rediscovering radicalism and humanity in social work. En M. Lavalette, (ed.). Radical social work today. Social work at the crossroads (pp. 153-163). Bristol: The Policy Press.

Lavalette, M. y Iokimidis, V. (2011). International social work or social work internationalism? Radical social work in global perspective. En M. Lavalette (ed.). Radical social work today. Social work at the crossroads (pp. 135-151). The Policy Press: Bristol.

Legault, G. (1997). Social Work Practice in Situations of Intercultural Misunderstandings. Journal of Multicultural Social Work, 4(4), 49-66. DOI: 10.1300/J285v04n04_04.

Lévesque, J. A. (2004). L'anthropopédagogie: une méthodologie au service d'un modèle de développement du jugement réflexif, Recherche-action, recherche systémique? Questions vives, État de la recherche en éducation, 2(3), 123-132. 
Lévesque, J. A. (2006). Training social workers in intercultural realities: A teaching model to counter unreasoned affectivity and contribute to the development of reflective judgment. En F. Pons et. al. (eds.). Towards Emotional Competences (pp. 229-248). Aalborg: Aalborg University Press.

Lévinas, E. (1991). Ética e infinito. Madrid: Machado Libros.

Maalouf, A. (1998). Identidades asesinas. Madrid: Alianza.

Marcus, G. y Fischer, M. (1986). La antropología como crítica cultural. Un momento experimental en las ciencias humanas. Buenos Aires: Amorrortu.

Novak, J. y Van Ewijk, H. (2010). From assimilation to intercultural competences: a challenge for social work. En H. Van Ewijk, European Social Policy and Social Work. Citizenship-based social work (pp. 130-139). Nueva York: Routledge.

Núñez, C. (1989). Educar para transformar, transformar para educar. San José: Alforja.

Okitikpi, T. y Aymer, C. (2010). Key Concepts in Anti-discriminatory Social Work. Londres: Sage.

Payne, M. y Askeland, G. A. (2008). Globalization and international social work: postmodern change and challenge. Aldershot: Ashgate.

Rivera, A.M. (2009). Regole e roghi. Metamorfosi del razzismo. Bari: Dedalo.

Ronnau, J. P. (1994). Teaching Cultural Competence: Practical Ideas for Social Work Educators. Journal of Multicultural Social Work, 3(1), 29-42. DOI: 10.1300/J285v03n01_04.

Rosaldo, R. (1993). Culture \& Truth: The Remaking of Social Analysis. Boston: Beacon Press.

Rose, S.M. y Black, B.I. (1985). Advocacy and Empowerment: Mental Health Care in the Community. Boston: Routledge \& Kegan Paul.

Sclavi, M. (2003). Arte di escoltare e mondi possibili. Milano: Bruno Mondadori.

Solomon, B. (1976). Black Empowerment: Social Work in Oppressed Communities. Nueva York: Columbia University Press.

Van Dijk, T. (2000). New(s) Racism. En S. Cottle (ed.) Ethnic minorities and the media (pp. 33-49). Buckingham: Open University Press.

Verbunt, G. (1994). Les obstacles culturels aux apprentissages. Paris: Centre National de Documentation Pédagogique.

Verbunt, G. (1999). Le problème des migrants et la formation des travailleurs sociaux. En J. Demorgon y E.M. Lipinasky (dir.). Guide de l'interculturel en formation (pp. 35-41). París: Retz.

Verbunt, G. (2004). La question interculturelle dans le travail social. Repères et perspectives. París: La Découverte.

Watzlawick P., Beavin, J., y Jackson, D. (1967). Teoría de la comunicación humana. Buenos Aires: Tiempo Contemporáneo. 